\title{
Systematic scrotum ultrasound in male infertility: what to look for
}

\author{
Ana Falticeanu, Olivier Lebecque, Michaël Dupont
}

Université catholique de Louvain, CHU UCL Namur, Department of Radiology, Yvoir, Belgium

\begin{abstract}
Ultrasound is a useful, cost-effective and minimally invasive tool that can be used in the workup of male infertility. Patient history, semen analysis and hormonal results often precede ultrasound examination as a part of the workup of male factor infertility. In our article, we advocate the added value of a systematic approach of the scrotal ultrasound. We propose a checklist for the complete analysis of testicular and paratesticular structures, useful in everyday practice for both clinicians and radiologists, highlighting what can be expected of and what should be found in the radiologist's report.

Keywords: infertility; ultrasound; scrotum; male; testis
\end{abstract}

\section{Introduction}

Imaging has become a major adjunct to clinical and laboratory examinations for the diagnosis and treatment of infertility. In the workup of infertility, a component factor of male infertility has been documented in about $40 \%$ of all infertile couples, whereas in $10-20 \%$ of cases male infertility is the sole identified factor [1-3] .

The most common etiologies of male infertility are idiopathic (33\%), varicoceles, varying up to $27 \%$ and obstructive causes $(15 \%)$, but also surgical or infectious sequelae, acquired anatomical problems, changes after radio-chemotherapy, as well as environmental toxins [3]. A vast majority of these conditions will have suggestive ultrasound appearance.

Usual evaluation of male infertility involves scrotal ultrasound which plays a crucial role besides medical history, medical examination and semen analysis [European Association of Urology (EAU) Guidelines 2016].

Received 03.03.2020 Accepted 20.04.2020

Med Ultrason

2020, Vol. 22, No 3, 364-369

Corresponding author: Ana Falticeanu

Université catholique de Louvain,

CHU UCL Namur, Department of Radiology

1 Avenue Dr G Thérasse, 5530, Yvoir, Belgium

E-mail: ana.falticeanu@uclouvain.be

Phone: $+32(0) 81423533$

Fax: $+32(0) 81423505$
Aiming at drafting a systematic approach on a standard ultrasonographic (US) examination, we will review what should be checked when performing scrotal ultrasound and their significance in this setting.

\section{Systematic scrotum ultrasound}

US is a helpful additional tool for diagnostic purposes, with the added value of being cost effective and minimally invasive. It is particularly useful in patients with a difficult clinical examination - such as obese patients, thickened scrotal wall or contracted scrotum.

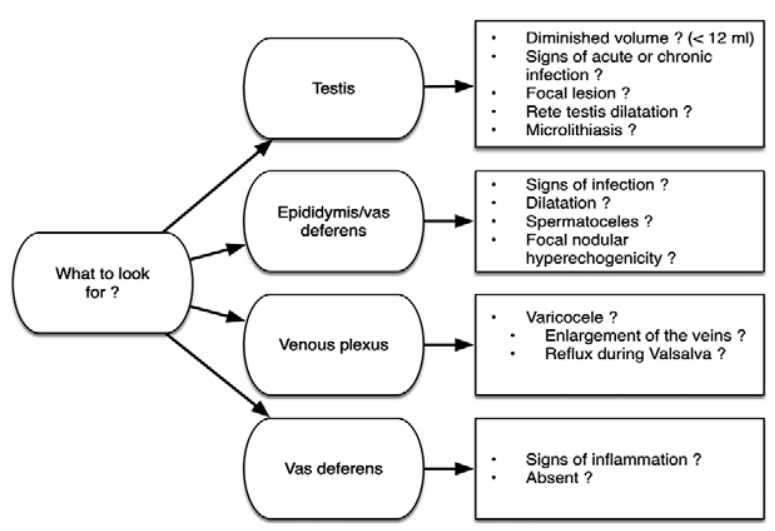

Fig 1. Male infertility ultrasound: what to look for - checkpoint system proposed by the authors. 
A systematic approach will ensure a complete analysis of the testicular and peritesticular structures as proposed in Figure 1. Testicular parenchyma, epididymis and spermatic cord should be analysed and colour Doppler used to evaluate blood flow within the testis and to determine the presence of varicocele.

The patient should be examined in a supine position with the scrotum properly supported. The highest frequency linear array transducer should be used that allows for proper penetration of the scrotum. Explaining the test to the patient will help to put them at ease. Warm gel should be used, and the examiner should test that the gel is not too hot.

\section{Testicular analysis}

\section{Volume}

Testicular volume is an important indicator of fertility. Multiple studies have shown that sperm density, total sperm count and total motile sperm count results correlated with a decreased testicular volume, especially in testes with a volume less than $10 \mathrm{ml}[3,4]$. Ultrasound has proven to be a better method of volume measurement than the traditional Prader orchidometer, the latter slightly overestimating volume, especially in small testes [5].

During the US workup, the standard measurements of length, width, and height should be obtained in the 3 orthogonal planes. The volume is then calculated using the formula for prolate ellipsoid. A normal volume is in the range of $15 \mathrm{~mL}-25 \mathrm{~mL}$, whereas a measure of less than $12 \mathrm{~mL}$ indicates a reduced testicular volume [6]. These measurements are particularly important in teens, as testicular atrophy associated with varicoceles is the most common indication for prophylactic repair in this age group. Varicocele, at this age, is proven to be associated with testicular atrophy and may impair fertility in adulthood $[5,7,8]$.

Testicular volume is an important parameter to assess especially in cases of obstructive etiologies. Volume is found to be higher in patients with obstructive azoospermia, with a mean volume $18.2 \mathrm{ml}$, versus patients with non-obstructive azoospermia with a mean volume $8.2 \mathrm{ml}[9,10]$, making this measurement particularly sensitive, as sometimes men with obstructive causes will undergo microsurgical reconstruction of the reproductive tract. Testicular dimensions should thus be reported as accurately as possible, with three orthogonal diameters and calculated volume for each testis.

\section{Parenchyma}

The specific analysis of the testicular parenchyma could reveal the presence of different anomalies: microlithiasis, sequelae of inflammation/infection or various focal lesions.

Testicular microlithiasis is an uncommon condition with an unknown aetiology that is detected incidentally by Ultrasound. The incidence widely varies, reported from 0.6 to $18 \%$ in various testicular US studies [9], [1113]. The diagnosis of testicular microlithiasis is made when five or more multiple small echogenic (less than $2 \mathrm{~mm}$ diameter each) and non-shadowing foci of a uniform size, which correspond to intratubular calcifications, are scattered throughout the parenchyma which keeps a normal shape, $[13,14]$. A grading system has been proposed taking into account the number of microcalcifications seen per field of view: grade I : mild testicular microlithiasis, between 5 and 10 calcifications per field; grade II - moderate, between 10 and 20 microlithiasis per field, and grade III - severe, more than 20 per field of view $[11,12]$ - example in figure 2 .

The relationship between the presence of microcalcifications and infertility is still unclear, but highly suspected. The association of testicular microlithiasis and testicular cancer has also been reported, but there is no clear consensus regarding the need for further evaluation or follow-up criteria. In infertile men, the presence of testicular microlithiasis has been associated with an approximated sevenfold higher cancer risk as compared
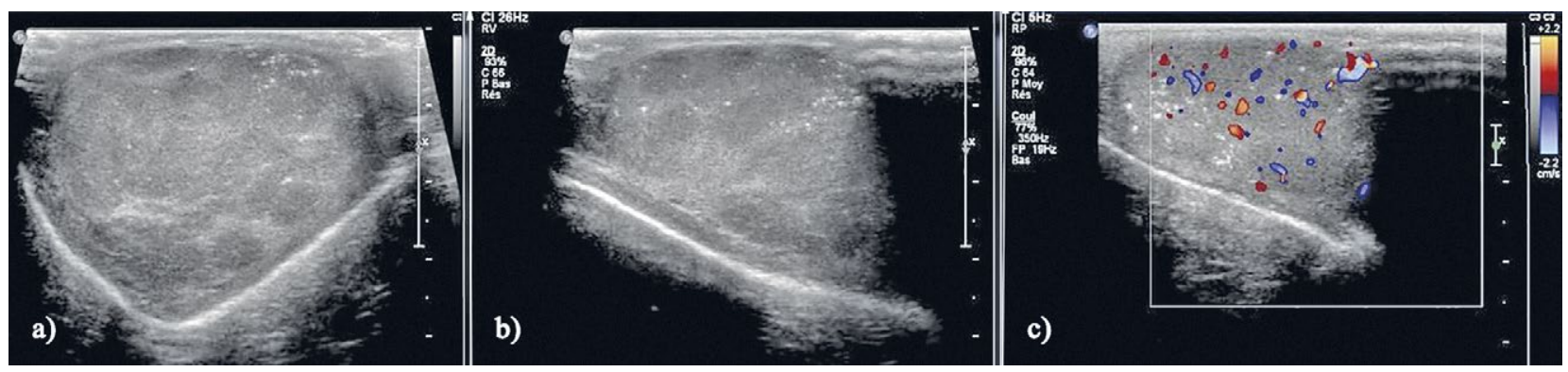

Fig 2. Focal microlithiasis in young male. Multiple microlithiasis associated with multifocal hypoechoic lesion (pathology confirmed testicular seminoma): a. transverse plane of the testis showing heterogeneous parenchyma; $b$. longitudinal plane of the testis showing heterogeneous parenchyma; c. longitudinal plane of the testis with Doppler colour showing hypervascularity within the hypoechoic lesion. 
to infertile men without microlithiasis $[13,15]$. Testicular microlithiasis, infertility and testicular cancer therefore seem to be interlinked, despite absence of uniform protocols for necessity, duration and interval of follow-up $[9,11]$. For patients with a high risk of developing testicular cancer, such as patients with cryptorchidism, infertility, testicular atrophy or contralateral testicular cancer, further evaluation is usually advised $[11,12,16]$.

Various other focal anomalies are picked up by US examinations and are commonly benign, but occasionally are of a malignant nature. Such anomalies occasionally encountered in the screened population are sequelae of previous infection or inflammation. While the acute episodes of orchitis, often associated with epididymitis [1], can present with a multitude of different features (hypoechoic regions with hyper-vascularity on Doppler, focal or diffuse, parenchyma swelling or scrotal wall thickening), with time chronic changes such as scarring or volume loss may be seen on US. Retraction of the testicular parenchyma can present as hypoechoic bands alternating with normal testicular parenchyma, although this finding is not specific only to orchitis sequelae.

Testicular neoplasms are known to be more frequent in infertile men and represent a cause of male infertility in up to $6 \%$ of cases. Most nodular lesions discovered by ultrasound in a population of infertile men are benign tumours and very few have specific identifying features [11].

In obstructive aetiologies, a different category of signs will be apparent, such as spermatocele, which can be difficult to differentiate from a simple cyst, and rete testis ectasia [17,18]. The latter should be differentiated from an intra-testicular varicocele using Doppler analysis, as they have the same grayscale appearance: linear hypo- or anechoic channels clustered in the same testicular region, showing important posterior acoustic enhancement (Figure 3). A distinctive trait could be the intra-testicular distribution: rete testis ectasia will always arise close to the testis mediastinum, whereas the intratesticular varicoceles can arise along the entire testicular parenchyma [19].

The difference between rete testis ectasia and cystic transformation of rete testis is not clear and often confounded in literature. It is however certain that testis cystic dysplasia is sometimes associated with renal agenesis or urogenital tract malformations [10,19-21]. Its presence should prompt further ultrasound investigations of the urinary tract. Although renal ultrasound is not commonly used in the diagnosis or treatment of male factor infertility, the embryologic origins and relations of metanephric system and mesonephros justifies renal US when congenital anomalies are suspected [11].

Absence of the epididymis and vas deferens can be demonstrated by scrotal US, and absence of ejaculatory duct or seminal vesicle by TRUS; these anomalies should prompt further workup with renal US.

\section{Epididymis}

The normal epididymis has an elongated shape and is seen as a discrete hypoechoic structure when compared to adjacent testicular parenchyma. It should be evaluated along its full length: initial segment, caput, corpus and caudal regions. Proximally, the efferent ducts connect the testis to the epididymis and distally, the vas deferens extends from the caudal region. The convoluted form of the vas deferens becomes more visible if there is inflammation as the walls become thicker and more hypo echoic.

A complete US protocol in the workup of male infertility includes careful epididymal analysis. Changes that can be seen within the epididymal parenchyma result from sequelae of acute conditions or obstructive pathology. The US classical features of acute epididymitis (hypoechoic aspect of the whole epididymis structure with

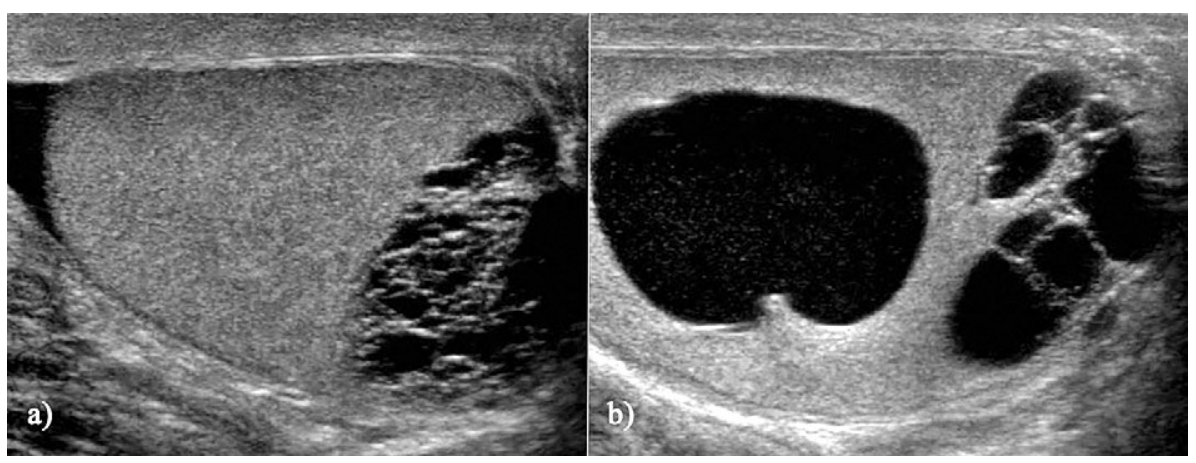

Fig 3. Rete testis ectasia in an elderly patient. Fluid filled serpiginous images without Doppler signal, associated with an intratesticular cystic lesion, presenting with internal echoes and irregular walls (spermatocele): a. oblique plane of the testis showing rete testis ectasia; b. longitudinal plane showing the rete testis ectasia and an associated spermatocele. 


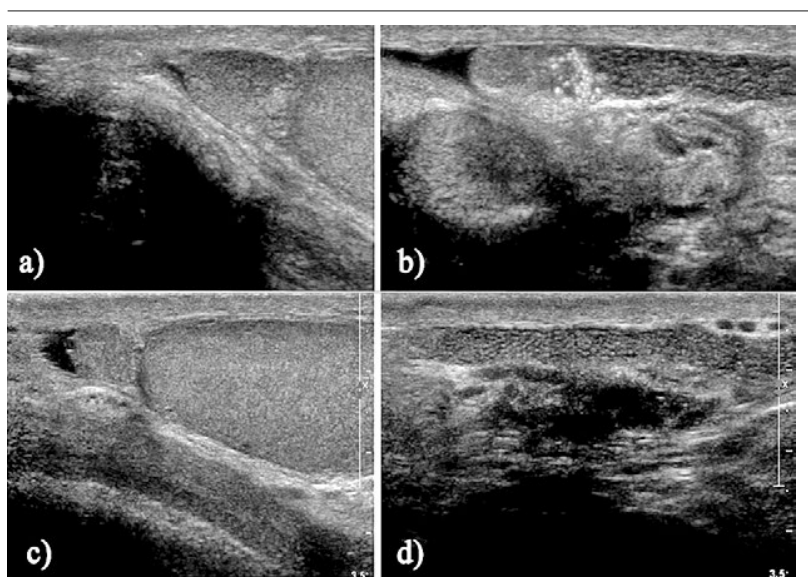

Fig 4. Bilateral epididymal tubular ectasia in an elderly patient. Upper a. normal epididymal cranial region; b. microcalcifications in the middle segment and tubular ectasia upstream; Lower c. normal epididymal cranial region d. tubular ectasia all along the corporeal and caudal regions.

sometimes marked hypervascularity) could resolve without any permanent changes, but changes in number and quality of sperm may be induced over time, mainly due to the spread to the testis in case of epididymo-orchitis (in a little over $20 \%$ of cases) $[2,12]$.

In the case of obstructive conditions, US may reveal an enlarged epididymis, often heterogeneous with tubular ectasia giving it a "sponge-like" appearance, and sometimes the presence of calcifications, such as in Figure 4. There are various aetiologies of epididymal obstruction, such as infectious diseases (especially tuberculosis), iatrogenic injuries and idiopathic obstructions.

Simple epididymal cysts are quite common in healthy young males, especially in the head of epididymis, and their isolated presence does not prompt further evaluation, in absence of relevant clinical context. Their primary differential diagnosis, just like in the testis, is a spermatocele, which is a fluid collection within the epididymal structures that contains spermatozoa and debris, and that may present with internal echoes and irregular internal walls. They will always arise from the head of the epididymis, in contrast to simple cysts that can arise throughout the length of the epididymis.

\section{Spermatic cord}

The spermatic cord can be found superior, posterior and lateral to the testis, and contains the ductus or vas deferens, the testicular artery and the pampiniform plexus of testicular veins.

\section{Vas deferens}

Ultrasound provides a non-invasive and real-time assessment particularly helpful in oligospermia and azoo- spermia patients. In obstructive conditions situated at the level of the spermatic cord, changes are very similar to an epididymal obstruction, mainly tubular ectasia sometimes accompanied by an increase in epididymal volume (calcifications may also be seen but they are not a consistent finding). Causes of obstruction may vary traumatic, iatrogenic (like vasectomy) or it can be related to the congenital absence or occlusion of the vas deferens, like in Zinner syndrome. As previously discussed, absence of Wolffian structures constitute one of the most frequent congenital abnormalities leading to infertility, and it has been proven to be linked to at least one mutation within the cystic fibrotic gene [9]. CFTR gene testing is indicated in patients diagnosed with unilateral or bilateral congenital absence of vas deferens. As some patients have other genitourinary anomalies, an abdominal ultrasound is also indicated $[11,20]$.

If the site of obstruction is situated at the level of the ejaculatory ducts the scrotal US findings may be similar, but usually the diagnosis relies on TRUS and shall not be detailed in this article.

\section{Varicoceles}

Varicocele represents dilation of the spermatic veins, serpiginous fluid filled structures slightly larger than normal venous calibre, located within the pampiniform plexus or in a peritesticular position. The left sided varicoceles are more frequent due to the different anatomy of the left spermatic vein compared to the right one, but up to $80 \%$ have been found to be bilateral in some studies $[11,13]$. The main mechanisms believed to explain the diminished fertility in the case of varicoceles includes increased levels of temperature of the testis during spermatogenesis and changes in arterial hydrostatic pressure, leading subsequently to hypoxia and oxidative stress $[6,7,11]$.

The systematic research for varicoceles is particularly important since it is the most common reversible cause of male infertility. It has been shown that its treatment may reverse testicular dysfunction and improve sperm production and morphology, thus increasing chances of pregnancy. Without US, physical examination alone can miss about $10 \%$ of left-sided and $90 \%$ of right-sided varicoceles. A study of 156 men found that the spermatic vein becomes palpable only at 3-3.5 mm [14]. Patients with subclinical varicoceles are thus the ones who will mostly benefit from the US examination.

For the ultrasound study the patients should be examined in both supine and then upright position, after a brief explanation of how to perform the Valsalva maneuver correctly. Classical longitudinal and axial planes are used to assess the area with the highest venous diameters, exploring the entire area from the level of the inguinal 


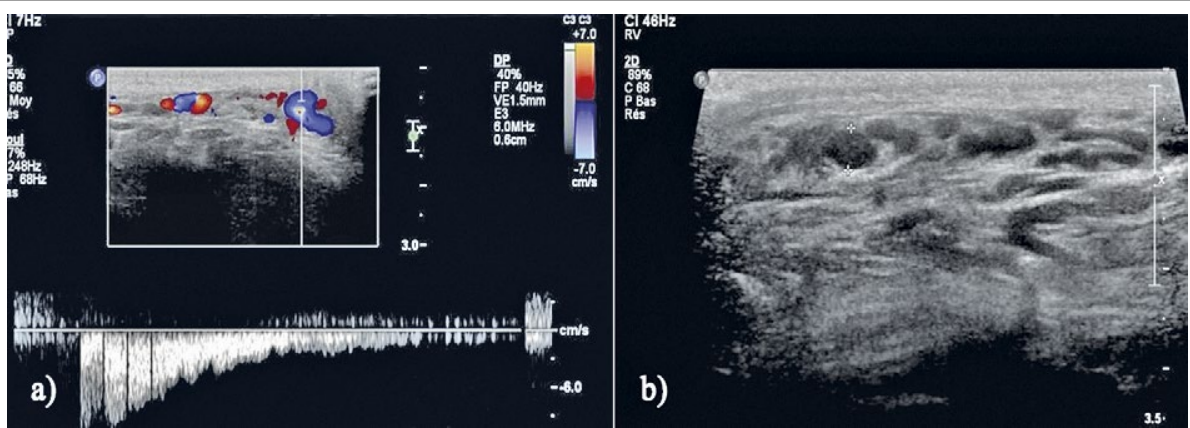

Fig 5. Varicoceles in a 34-year-old man: a. induced reversal of blood flow during Valsalva maneuver; b. abnormally dilated veins in the pampiniform plexus next to the cranial region of the testis

canal to the inferior pole of the testis. The presence of venous reflux is evaluated before and during execution of the Valsalva manoeuvre (Figure 5). The duration of the reflux, rather than its velocity, is evaluated, so the steering angle is not normally used. Obviously, the operator should take caution to avoid exerting excessive pressure with the transducer.

For the diagnosis of varicoceles the maximum venous diameter is analysed: a diameter of more than $2.95 \mathrm{~mm}$ during Valsalva has been associated with $84 \%$ of sensitivity and specificity for clinical varicoceles $[9,22,23]$; in current practice a cut-off of $3 \mathrm{~mm}$ is easier to use. As for the duration of the reflux - more than 1 second is considered positive for varicoceles during Valsalva manoeuvre. If there is flow reversal (or reflux), varicoceles repair can improve sperm parameters for patients who had peritesticular veins with a diameter larger than $3 \mathrm{~mm}$ $[3,24]$.

The most used grading classification in the literature is the Sarteschi classification [23], although it is less used in common practice. A simpler and more useful description that could be used is the level at which the dilated venous structures are seen: at the level of the pampiniform plexus or in a peritesticular position: at the level of the superior/ middle/inferior third of the testis.

\section{Conclusion}

Male infertility is a complex phenomenon for which different etiologies may be the cause. Ultrasound evaluation is cost effective and minimally invasive, representing an excellent tool for the initial workup when used in adjunction to a thorough medical history, clinical examination, and semen and hormone analysis.

We advocate the added value of a systematic approach of the scrotal ultrasound and we propose a checkpoint system useful in everyday practice. Cut-off values should be used when describing testicular volume, microlithiasis and/or varicocele.
A methodical analysis includes testicular evaluation - dimensions, presence of focal lesions within the testicular parenchyma with a special focus on microlithiasis, followed by analysis of peritesticular structures: the epididymis and spermatic cord. Special attention should be given to the presence of varicocele, a common condition that should be screened for routinely during the scrotal ultrasound work-up for infertility.

\section{Conflict of interest: none}

\section{Bibliography}

1. Ammar T, Sidhu PS, Wilkins CJ. Male infertility: the role of imaging in diagnosis and management. BJR. 2012;85(special_issue_1):S59-S68

2. Mittal PK, Little B, Harri PA, et al. Role of Imaging in the Evaluation of Male Infertility. RadioGraphics. 2017;37(3):837-854.

3. Armstrong JM, Keihani S, Hotaling JM. Use of Ultrasound in Male Infertility: Appropriate Selection of Men for Scrotal Ultrasound. Curr Urol Rep. 2018;19(8):58.

4. Sakamoto H, Saito K, Ogawa Y, Yoshida H. Testicular Volume Measurements Using Prader Orchidometer Versus Ultrasonography in Patients with Infertility. Urology. 2007;69(1):158-162.

5. Sakamoto H, Ogawa Y. Does a clinical varicocele influence the relationship between testicular volume by ultrasound and testicular function in patients with infertility? Fertility and Sterility. 2009;92(5):1632-1637.

6. Ali JI, Weaver DJ, Weinstein SH, Grimes EM. Scrotal Temperature and Semen Quality in Men With and Without Varicocele. Archives of Andrology. 1990;24(2):215-219.

7. Zorgniotti AW, MacLEOD J. Studies in temperature, human semen quality, and varicocele. Obstetrical \& Gynecological Survey. 1974;29(8):567-568.

8. Condorelli R, Calogero AE, La Vignera S. Relationship between Testicular Volume and Conventional or Nonconventional Sperm Parameters. International Journal of Endocrinology. 2013;2013:1-6.

9. Sihag P, Tandon A, Pal R, et al. Sonography in male infertility: a look beyond the obvious. J Ultrasound. 2018;21(3):265-276. 
10. Abdulwahed SR, Mohamed E-EM, Taha EA, Saleh MA, Abdelsalam YM, ElGanainy EO. Sensitivity and Specificity of Ultrasonography in Predicting Etiology of Azoospermia. Urology. 2013;81(5):967-971.

11. Kekre N, Shanmugasundaram R, Singh Jc. Testicular microlithiasis: Is there an agreed protocol? Indian J Urol. 2007;23(3):234.

12. Miller FNAC, Sidhu PS. Does Testicular Microlithiasis Matter? A Review. Clinical Radiology. 2002;57(10):883890.

13. Heller HT, Oliff MC, Doubilet PM, O’Leary MP, Benson CB. Testicular microlithiasis: Prevalence and association with primary testicular neoplasm: Testicular Microlithiasis. J Clin Ultrasound. 2014;42(7):423-426.

14. Pedersen MR, Rafaelsen SR, Møller H, Vedsted P, Osther PJ. Testicular microlithiasis and testicular cancer: review of the literature. Int Urol Nephrol. 2016;48(7):1079-1086.

15. Aso C, Enríquez G, Fité M, et al. Gray-Scale and Color Doppler Sonography of Scrotal Disorders in Children: An Update. RadioGraphics. 2005;25(5):1197-1214.

16. Kuligowska E, Fenlon HM. Transrectal US in male infertility: spectrum of findings and role in patient care. Radiology. 1998;207(1):173-181.

17. Dogra VS, Gottlieb RH, Rubens DJ, Liao L. Benign Intratesticular Cystic Lesions: US Features. RadioGraphics. 2001;21(suppl_1):S273-S281.
18. Mahlknecht A, Mahlknecht P, Fallaha M, Wieser A. Tubular ectasia of the rete testis (TERT). Differential diagnosis of cystic testicular disorders. Arch Ital Urol Androl. 2015;87(1):5.

19. Lane VA, Scammell S, West N, Murthi GV. Congenital absence of the vas deferens and unilateral renal agenesis: implications for patient and family. Pediatr Surg Int. 2014;30(7):733-736.

20. Kara T, Durmaz MS, Ceken K. Ultrasonography of tubular ectasia of the rete testis with associated scrotal findings. J Med Ultrasonics. 2013;40(1):27-32.

21. Hoekstra T, Witt MA. The Correlation of Internal Spermatic Vein Palpability With Ultrasonographic Diameter and Reversal of Venous Flow. Journal of Urology. 1995;153(1):8284.

22. Pilatz A, Altinkilic B, Köhler E, Marconi M, Weidner W. Color Doppler ultrasound imaging in varicoceles: is the venous diameter sufficient for predicting clinical and subclinical varicocele? World J Urol. 2011;29(5):645-650.

23. Iosa G, Lazzarini D. Hemodynamic classification of varicoceles in men: our experience. J Ultrasound. 2013;16(2):5763.

24. Schiff JD, Li PS, Goldstein M. Correlation of ultrasoundmeasured venous size and reversal of flow with Valsalva with improvement in semen-analysis parameters after varicocelectomy. Fertility and Sterility. 2006;86(1):250-252. 